\title{
Quality of Life (QoL) of People with Spinal Cord Injury (SCl) in Western Greece
}

\author{
Ioannis-Alexandros Tzanos ${ }^{1,}$, , Athanasios Kyriakides ${ }^{1}$, Evgenia Gkintoni ${ }^{2}$, Elias Panagiotopoulos ${ }^{1}$ \\ ${ }^{1}$ Rehabilitation Department, University of Patras, Patras, Greece \\ ${ }^{2}$ Psychology Department, University Hospital of Patras, Patras, Greece
}

Email address:

tzanosalexandros@yahoo.gr (Ioannis-Alexandros T.)

${ }^{*}$ Corresponding author

\section{To cite this article:}

Ioannis-Alexandros Tzanos, Athanasios Kyriakides, Evgenia Gkintoni, Elias Panagiotopoulos. Quality of Life (QoL) of People with Spinal Cord Injury (SCI) in Western Greece. Rehabilitation Science. Vol. 4, No. 1, 2019, pp. 7-12. doi: 10.11648/j.rs.20190401.12

Received: June 24, 2019; Accepted: July 15, 2019; Published: July 31, 2019

\begin{abstract}
Community living individuals with SCI face major health-related QoL challenges, especially in countries such as Greece, due to the lack of infrastructure and support networks. The aim of this study is to evaluate QoL of people with SCI who have already completed the rehabilitation program and live in the geographic area of Western Greece and to identify the clinical and demographic factors that affect QoL in this population. Thirty-six individuals with SCI living in Western Greece participated in this study. We used World Health Organization Quality of Life BREF (WHOQOL-BREF) questionnaire to estimate their QoL levels. Additional data were collected by clinical measurements and a demographic questionnaire. The mean age of the sample was $45.1 \pm 9.8$ years, the mean time since injury was $13.1 \pm 11.7$ years and $89 \%$ of the participants were male. The mean values of QoL parameters ranged from 56.6 \pm 16.6 up to 100 (physical health) to $67.7 \pm 18.1$ up to 100 (environment). Lower education level $(\mathrm{p}=0.035)$ and married life $(\mathrm{p}=0.006)$ are associated with higher QoL levels regarding environment and social relationships respectively. Additionally, as time since injury passes, the aspect of Qol that is related with social relationships improves $(\mathrm{p}=0.04)$. The study documents relatively high rates of QoL in the population of individuals with SCI in Western Greece. Married life, low educational level and long time post injury are determinants of high QoL levels in this population.
\end{abstract}

Keywords: Spinal Cord Injury, Quality of Life, Physical Activity, Pressure Ulcers, Urinary Incontinence

\section{Introduction}

Spinal cord injury is a severe medical condition, which may result in paralysis, sensory deficits and sphincter dysfunction [1]. People with acquired SCI, after completing the inpatient rehabilitation program and enter community life, they face major health-related QoL challenges, regarding well-being, social participation and ability to perform activities of daily life [2]. The technology development during the last decades has promoted QoL of individuals with SCI - the wide use of computer assisted technology and the advances in motor devices have enhanced the ability of those people to have access in knowledge, education, and work, to travel and to participate in leisure activities [3].

Previous studies have identified the major factors that affect QoL of individuals with SCI in various countries and regions. Age, time passed since the injury, gender, marital status, level of injury, employment status, education level and presence of pain or pressure ulcers (PUs) are the main variables that have been thoroughly examined in literature [4-7]. There is a general consensus that short time since the injury, pain and PUs are the main predicting factors of low QoL levels in various populations of people with SCI [4-7].

However, every country has its own structural and cultural characteristics that differentiate the profile of challenges for persons with SCI. In Greece, there is a lack of policies for support and infrastructure for people with disabilities [8]. On the other hand, family and social networks play an important supportive role in an individual's life which compensates for these difficulties [9]. The aim of this cross-sectional study is to evaluate the levels of QoL of people with SCI that live in Western Greece and to highlight the major sociodemographic 
and clinical factors that tend to limit the QoL levels in this population.

\section{Methods}

\section{Participants, design and setting}

Thirty-six individuals with SCI of several causes living in the Prefecture of Western Greece cooperated for the implementation of the current scientific project. The recruitment of the sample was enhanced by having access to the medical archives of outpatients and previous inpatients of the Spinal Cord Injury Rehabilitation Department of the University Hospital of Patras which is the only SCI institution in Western Greece. The local association of people with SCI also collaborated with the research team in order to detect the whole SCI population of this region. Thirty-six out of fifty persons with SCI found in Western Greece approved to participate in this study. The collection of the data took place during the term from August 2018 to October 2018.

\section{Inclusion criteria}

We included in this study both male and female individuals with SCI that had already completed a rehabilitation program and had been living in the community for at least one year. We also included both paraplegic and tetraplegic persons with all classes of severity of SCI according to the American Spinal Injury Association (ASIA) classification. However, we excluded persons with SCI that presented with concurrent traumatic brain injury or with cognitive deficits of any reason. We also excluded people with SCI that were aged $<18$ years and those that were not fluent Greek language speakers.

Hypothesis

We hypothesized that QoL levels of individuals with SCI in Western Greece would be similar to those that were found in previous literature regarding the whole country. We also hypothesized that the presence of PUs and unemployment would be determinants of low levels of QoL in this population of people with SCI.

\section{Questionnaires}

World Health Organization Quality of Life BREF (WHOQOL-BREF) questionnaire was administered to all participants along with a demographic questionnaire in interview form and clinical evaluation. The WHOQOL-BREF scale was utilized in order to assess QoL. The WHOQOL project was initiated in 1991 in purpose of developing an international cross-culturally comparable QoL measurement tool [7]. It evaluates the individual's perceptions regarding their culture and principle systems, along with personal ambitions, standards and worries [10]. The WHOQOL-BREF is the brief version of the original tool. It consists of 26 items that assess four broad domains: physical health, psychological well-being, social relationships, and environment. It is generally approved as useful in large research projects or clinical trials [11, 12]. However, the demographic questionnaire included information concerning age, gender, marital status, place of residence, injury date, educational level and occupation profile. The clinical assessment provided information regarding the level of injury (paraplegia or tetraplegia), the existence of pressure ulcers, the report of urinary incontinence symptoms and the involvement of the participants in physical activity during the last six months. We also used the American Spinal Injury Association Impairment Scale (AIS), which is consistent with the ASIA neurological classification system, in order to evaluate the severity of the SCI [13]. Furthermore, we used the $0-10$ point pain scale to assess the intensity of pain of the respondents, asking them to self-grade their pain degree during the last week.

\section{Ethical issues}

This study had obtained approval from the University Hospital of Patras Ethics Committee. All participants that were consisted with the inclusion criteria were asked to participate after getting an explanation of the study's aims and information about being entitled to withdraw from the study whenever they want with no consequences. The participants were also asked to sign a consent form that fully complied with the rules of the Helsinki declaration and the Hellenic State Medical ethics legislation (Hellenic State Law No. 3418/2005). Thus, we confirm that all relevant institutional and administrative regulations regarding the ethical participation of human volunteers were adhered to during the setting of this scientific research.

\section{Statistical analysis}

The data is expressed as means \pm standard deviation (SD) for continuous parameters and as percentages for categorical variables. The normality of the distribution of the variables was measured with the Kolmogorov-Smirnov test and the normal probability plot. The bivariate analysis was performed using the Student's t-test in order to investigate the correlation between the dependent parameter (instrument's scores) and the qualitative variables. Pearson's correlation coefficient was utilized to explore the correlation between each dependent parameter and the quantitative variables. A p-value of $<0.05$ indicated statistical significance. All analyses were made utilizing the statistical package SPSS version 17.00 (Statistical Package for the Social Sciences, SPSS Inc., Chicago, Ill., USA).

\section{Results}

The mean age was $45.1 \pm 9.8$ years, the mean time since the initiation of the disability was $13.1 \pm 11.7$ years and $89 \%$ of the participants were male. They were also primarily graduates of primary or secondary school $(83.3 \%)$ and they resided mainly in urban territories $(72.2 \%)$. However, the majority of the participants were unmarried (61.1\%) and only the $16.7 \%$ of the sample was professionally active (students and working people). The sociodemographic characteristics of the sample are presented in Table 1.

The participants were mainly paraplegic $(83.3 \%)$ and with a motor complete injury, namely classified as A or B in AIS (63.9\%). They also mainly presented without PUs (83.3\%) and without reported symptoms of urinary incontinence $(58.3 \%)$. Furthermore, the majority of the sample $(72.2 \%)$ was involved in physical activity during the last six months (72.2\%). Moreover, the participants' pain score during the 
week prior the evaluation was $4.2 \pm 2.4$, measured according to the 0-10 point pain scale. The clinical features of the sample are presented in Table 2.

The mean values of QoL parameters in our sample according to WHOQOL-BREF scale were $56.6 \pm 16.6$ up to 100 (physical health), $65 \pm 18.2$ up to 100 (psychological), $65.5 \pm 24.8$ up to 100 (social relationships) and $67.7 \pm 18.1$ up to 100 (environment).

Gender, place of residence, occupation status, level of injury, AIS, presence of PUs or urinary incontinence, involvement in physical activity, age, and pain intensity had no statistically significant correlation with any of the four QoL domains of WHOQOL-BREF scale. On the other hand, married participants presented with higher WHOQOL-BREF scores regarding social relationships than the unmarried ones $(\mathrm{p}=0.006)$ and those who have graduated from tertiary education presented with lower WHOQOL-BREF scores regarding environment than those with lower education level $(p=0.035)$. Furthermore, as the participants were more years far apart the injury, they exhibited higher WHOQOL-BREF scores regarding social relationships $(\mathrm{r}=0.343, \mathrm{p}=0.04)$.

Table 1. Sociodemographic characteristics of the sample.

\begin{tabular}{|c|c|c|c|c|}
\hline & & & $\mathbf{N}$ & $\%$ \\
\hline \multirow{2}{*}{ Gender } & \multicolumn{2}{|l|}{ Male } & 32 & 88.9 \\
\hline & \multicolumn{2}{|c|}{ Female } & 4 & 11.1 \\
\hline \multirow{2}{*}{ Marital status } & \multicolumn{2}{|c|}{ Married } & 14 & 38.9 \\
\hline & \multicolumn{2}{|c|}{ Unmarried } & 22 & 61.1 \\
\hline \multirow{2}{*}{ Place of residence } & \multicolumn{2}{|c|}{ Urban } & 26 & 72.2 \\
\hline & \multicolumn{2}{|c|}{ Suburban/Rural } & 10 & 27.8 \\
\hline \multirow{2}{*}{ Educational level } & \multicolumn{2}{|c|}{ Primary/Secondary } & 30 & 83.3 \\
\hline & \multicolumn{2}{|c|}{ Tertiary } & 6 & 16.7 \\
\hline \multirow{3}{*}{ Occupation } & \multicolumn{2}{|c|}{ Unemployed/Pensioner } & 30 & 83.3 \\
\hline & \multicolumn{2}{|c|}{ Working/Student } & 6 & 16.7 \\
\hline & Mean & SD & Min & Max \\
\hline Age & 45.1 & 9.8 & 29 & 74 \\
\hline Years post Injury & 13.1 & 11.7 & 1 & 39 \\
\hline
\end{tabular}

Table 2. Clinical characteristics of the sample.

\begin{tabular}{|c|c|c|c|c|}
\hline & & & $\mathbf{N}$ & $\%$ \\
\hline \multirow{2}{*}{ Type of SCI } & \multicolumn{2}{|c|}{ Paraplegia } & 30 & 83.3 \\
\hline & \multicolumn{2}{|c|}{ Tetraplegia } & 6 & 16.7 \\
\hline \multirow{2}{*}{ AIS } & \multicolumn{2}{|c|}{ A, B (motor complete) } & 23 & 63.9 \\
\hline & \multicolumn{2}{|c|}{$\mathrm{C}, \mathrm{D}, \mathrm{E}$ (motor incomplete) } & 13 & 36.1 \\
\hline \multirow{2}{*}{ Pressure ulcers } & \multicolumn{2}{|c|}{ No } & 30 & 83.3 \\
\hline & \multicolumn{2}{|l|}{ Yes } & 6 & 16.7 \\
\hline \multirow{2}{*}{ Urinary incontinence } & \multicolumn{2}{|l|}{ No } & 21 & 58.3 \\
\hline & \multicolumn{2}{|l|}{ Yes } & 15 & 41.7 \\
\hline \multirow{3}{*}{ Physical activity } & \multirow{2}{*}{\multicolumn{2}{|c|}{$\begin{array}{l}\text { No } \\
\text { Yes }\end{array}$}} & 10 & 27.8 \\
\hline & & & 26 & 72.2 \\
\hline & Mean & SD & Min & Max \\
\hline Pain score & 4.2 & 2.4 & 0 & 10 \\
\hline
\end{tabular}

Table 3. Correlation of WHOQOL-BREF with qualitative measurements of the sample.

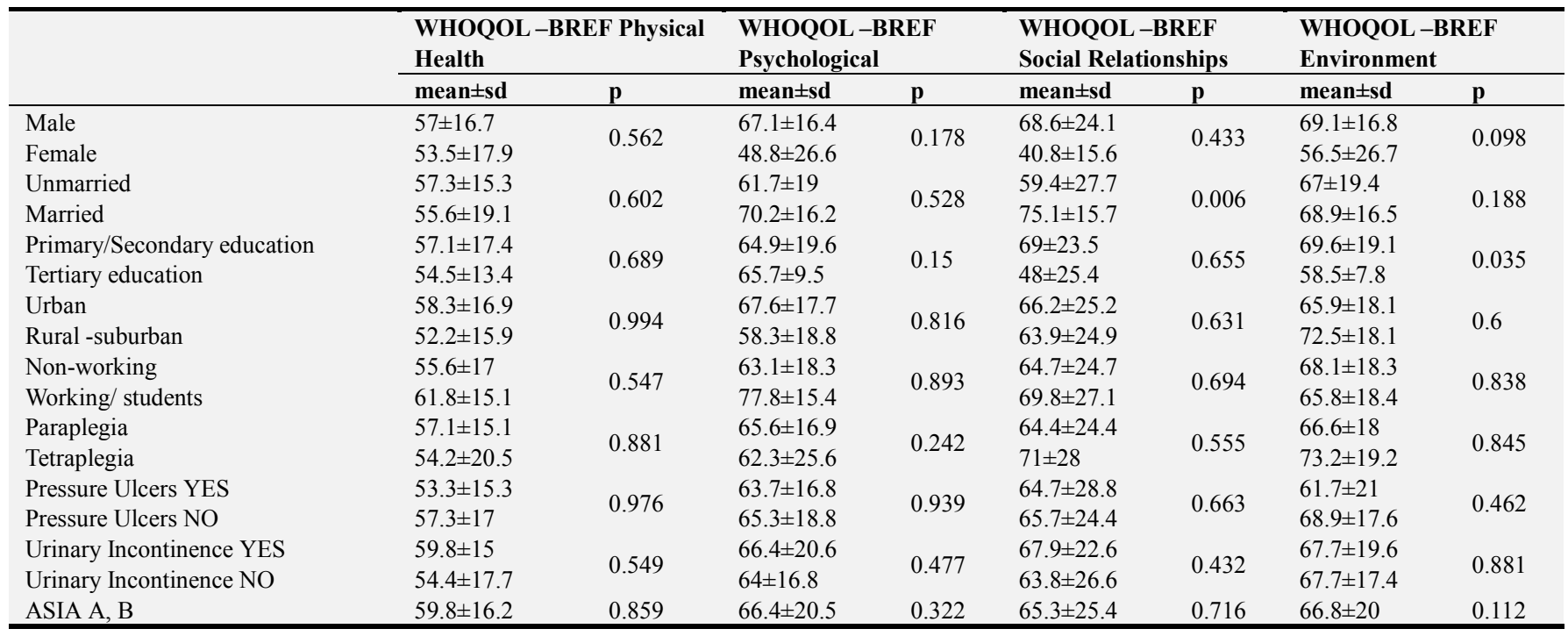




\begin{tabular}{|c|c|c|c|c|c|c|c|c|}
\hline & \multicolumn{2}{|c|}{$\begin{array}{l}\text { WHOQOL-BREF Physical } \\
\text { Health }\end{array}$} & \multicolumn{2}{|c|}{$\begin{array}{l}\text { WHOQOL -BREF } \\
\text { Psychological }\end{array}$} & \multicolumn{2}{|c|}{$\begin{array}{l}\text { WHOQOL-BREF } \\
\text { Social Relationships }\end{array}$} & \multicolumn{2}{|c|}{$\begin{array}{l}\text { WHOQOL-BREF } \\
\text { Environment }\end{array}$} \\
\hline & mean \pm sd & $\mathbf{p}$ & mean \pm sd & $\mathbf{p}$ & mean \pm sd & $\mathbf{p}$ & mean \pm sd & $\mathbf{p}$ \\
\hline ASIA C, D, E & $51 \pm 16.5$ & & $62.5 \pm 13.6$ & & $66 \pm 24.4$ & & $69.3 \pm 14.7$ & \\
\hline $\begin{array}{l}\text { Physical Activity YES } \\
\text { Physical Activity NO }\end{array}$ & $\begin{array}{l}57.7 \pm 15.8 \\
54 \pm 19.2\end{array}$ & 0.568 & $\begin{array}{l}68.1 \pm 14.5 \\
57.1 \pm 24.7\end{array}$ & 0.124 & $\begin{array}{l}67.8 \pm 24.7 \\
59.5 \pm 25.3\end{array}$ & 0.668 & $\begin{array}{l}69.4 \pm 16.7 \\
63.3 \pm 21.8\end{array}$ & 0.229 \\
\hline
\end{tabular}

Table 4. Correlation of WHOQOL-BREF with quantitative measurements of the sample.

\begin{tabular}{lllllllll}
\hline & \multicolumn{2}{l}{$\begin{array}{l}\text { WHOQOL - BREF Physical } \\
\text { Health }\end{array}$} & $\begin{array}{l}\text { WHOQOL - BREF } \\
\text { Psychological }\end{array}$ & \multicolumn{2}{l}{$\begin{array}{l}\text { WHOQOL - BREF Social } \\
\text { Relationships }\end{array}$} & $\begin{array}{l}\text { WHOQOL - BREF } \\
\text { Environment }\end{array}$ \\
\cline { 2 - 9 } & R & p & R & p & R & p & R & p \\
\hline Age & -0.144 & 0.402 & -0.127 & 0.460 & -0.069 & 0.688 & -0.038 & 0.824 \\
Years post SCI & 0.209 & 0.221 & 0.121 & 0.482 & 0.343 & $\mathbf{0 . 0 4}$ & 0.224 & 0.189 \\
Pain intensity & 0.284 & 0.093 & 0.268 & 0.114 & 0.186 & 0.277 & -0.013 & 0.940 \\
\hline
\end{tabular}

R: Pearson's correlation coefficient.

\section{Discussion}

The results of this study show that QoL levels in people with SCI in Western Greece are slightly higher than those reported in other European countries [4, 5]. However, we did not find any difference in comparison with results from the whole Greek territory according to previous data [9]. Although people with SCI in Western Greece face major limitations regarding mobility, accessibility (streets, buildings, transportation, etc) and work opportunities due to the lack of the essential policies [8], other factors such as the traditional family support network, the supporting role of church, the existence of a free access Public Health Care System, and the Greek culture, that is characterized by warm human relationships, are of greater significance [14]. Furthermore, the foundation and operation of the Rehabilitation Department for Patients with SCI during the last years provides high level medical, nursing and psychological surveillance and support. The Department has also empowered the formation of networks between people with SCI and it has enhanced their involvement in sports activities, promoting their social life and relationships [15].

Despite that previous studies have reported that women with SCI are characterized by decreased vitality and vigor [6], and with a higher rate of medical, social and psychological problems [16] compared with men, in our sample women and men showed equal levels of QoL. We also found that married people with SCI in this region exhibit greater QoL in the domain of social relations. This result is consistent with other studies that have reported that living with a spouse is associated with lower depression rates, better psychological well-being and increased social participation $[17,18]$.

Our findings regarding decreased QoL among people that have graduated from tertiary education could attributed to the fact that high education level is often associated with jobs with increased working stress. This is contrary to studies from other countries [19-22]. Specifically, it has been reported that education in patients with SCI enhances job finding, especially jobs with higher income, which provides access to goods and recreation [19], and improves confidence and self-efficacy [21]. Moreover, despite our findings, employment has been identified as having a positive effect on QoL, enhancing social relationships, interaction with community and adoption of productive social roles $[6,7]$. However, in our sample, only $16.7 \%$ of the participants declared having a job, which is a very low number considering that the mean age was 45 years. This finding could be partially explained by the high unemployment rate in Western Greece during data collection $(>20 \%)$ and by the existing benefit policies, that lead individuals with SCI to prefer retirement instead of continuing working or searching for a job.

Contrary to our results, age has been reported as a factor that affects QoL of people with SCI [5, 23]. More specifically, as age increases, it seems to deteriorate QoL by worsening health level, due to multiple co-morbidities and increasing fatigue and disability in the elderly [5, 23]. However, long time since the initiation of the disability had a beneficial effect in QoL in our sample. This is consistent with part of the literature $[6,19]$ and attributed to the improvement of psychology and self-perception of health state of people with SCI as time passes, while other authors did not find any significant correlation [23, 24]. Furthermore, there was no difference in QoL between residents of urban and rural areas in our sample. This is consistent with previous results from the whole country [9], while a study from Brazil found that residing with SCI in urban areas is associated with a greater level of satisfaction with life compared to those living in rural areas [25].

The level of injury, the severity of the injury according to AIS, the presence of PUs, the lack of physical activity, the occurrence of urinary incontinence and the intensity of pain were not significantly correlated with QoL levels in our sample. However, other authors have reported that paraplegic individuals exhibit higher QoL than tetraplegic individuals, which is partly attributed to their higher independence level, their lower mobility limitations and therefore higher ability to work and to participate in leisure and social activities [5, 23]. Furthermore, the presence of PUs has been previously associated with low QoL levels, having a negative impact on self-care, mobility, self-esteem [26], physical and community functioning [7], independence in activities of daily life [24], and emotional status [25]. Moreover, pain has also been considered as a predictor of low QoL levels in persons with SCI, as it negatively affects mood, sleep, self-care and 
mobility [27], it increases depression risk [28], it reduces satisfaction with life [28], and it restricts participation in recreation and leisure activities [27]. One the other hand, although Burke et al highlighted the significant and negative effect of pain, especially neuropathic pain and high intensity pain on QoL of people with acquired SCI, they identified simple pain existence as the most accurate predictor of low QoL levels regarding all the four domains of WHOQOLBREF scale rather than pain intensity or pain type (neuropathic or nociceptive pain [29].

\section{Study Limitations}

This study has various limitations. The size of our sample was relatively small and this resulted to a limited number of female and tetraplegic subjects. This could mean that it is disputable that the target population is represented accurately by our sample. In addition, the enrollment was voluntary and this could have an impact on the medical and demographic characteristics of the sample that limits the generalization of our findings. Another limitation of the study is the self-report method to evaluate pain which may be influenced by other variables such as depression.

\section{Conclusions}

This study documents similar to relatively high rates of QoL in the population of individuals with SCI in Western Greece in comparison with other countries and approximately similar rates with the rest of Greek territory. This could be interpreted by the easy and well-organized access to health services (good out-of-hospital follow up) and the steady medical surveillance by physiatrists in the SCI rehabilitation department of the University Hospital of Patras. Married life, low education level and long time since the initiation of the disability are determinants of high levels of QoL among people with SCI in this region.

\section{Financial Support and Sponsorship}

This research is co-financed by Greece and the European Union (European Social Fund- ESF) through the Operational Programme "Human Resources Development, Education and Lifelong Learning 2014-2020" in the context of the project "Effectiveness of holistic care in the quality of life of patients with Spinal Cord Injury (SCI)" (MIS 5005958).

\section{Conflicts of Interest}

There are no conflicts of interest.

\section{Abbreviations}

$$
\begin{array}{ll}
\text { SCI } & \text { Spinal cord injury } \\
\text { QoL } & \text { Quality of Life }
\end{array}
$$$$
\text { WHOQOL World Health Organization Quality of Life }
$$$$
\text { PUs Pressure ulcers }
$$

$\begin{array}{ll}\text { ASIA } & \begin{array}{l}\text { American Spinal Injury Association } \\ \text { American Spinal Injury Association }\end{array} \\ \text { AIS } & \text { Impairment Scale }\end{array}$

\section{References}

[1] Holtz A, Levi R. Spinal Cord Injury. Oxford University Press. 6 July 2010.

[2] Boakye, M., \& Barbara, C. L. S. A. (2012). Quality of life in persons with spinal cord injury: Comparisons with other populations. Journal of Neurosurgery Spine, 17 (September), 29-37.

[3] Cooper RA, Cooper R. Quality-of-life technology for people with spinal cord injuries. Phys Med Rehabil Clin N Am. 2010 Feb; 21 (1): 1-13.

[4] Kennedy P, Lude P, Taylor N. Quality of life, social participation, appraisals and coping post spinal cord injury: a review of four community samples. Spinal Cord. 2006; 44: 95-105.

[5] Fekete C, Wahrendorf M, Reinhardt JD, Post MWM, Siegrist J. Work stress and quality of life in persons with disabilities from four European countries: the case of spinal cord injury. Qual Life Res. 2014; 3: 1661-71.

[6] Westgren N, Levi R. Quality of life and traumatic spinal cord injury. Arch Phys Med Rehabil. 1998; 79: 1433-9.

[7] Ottomanelli L, Barnett SD, Goetz LL. A prospective examination of the impact of a supported employment program and employment on health-related quality of life, handicap, and disability among Veterans with SCI. Qual Life Res. 2013; 22 (8): 2133-41.

[8] Rapidi CA, Kyriakides A. People with Spinal Cord Injury in Greece. American journal of physical medicine \& rehabilitation. Feb 2017; 96: S71-73.

[9] Ioannis-Alexandros Tzanos, Evanthia Mitsiokapa, Panayiotis D. Megaloikonomos, Vasileios G. Igoumenou, Georgios N. Panagopoulos, Jannis Papathanasiou, George Tzanos, Andreas F. Mavrogenis. Social Reintegration and Quality of Life after Spinal Cord Injury: the Greek Paradigm. Journal of Biomedicine 2016; 1: 36-43.

[10] Study protocol for the World Health Organization project to develop a Quality of Life assessment instrument (WHOQOL). Qual Life Res. 1993; 2 (2): 153-9.

[11] Jang Y, Hsieh CL, Wang YH, Wu YH. A validity study of the WHOQOL-BREF assessment in persons with traumatic spinal cord injury. Arch Phys Med Rehabil. 2004; 85 (11): 1890-5.

[12] Lin MR, Hwang HF, Chen CY, Chiu WT. Comparisons of the brief form of the World Health Organization Quality of Life and Short Form-36 for persons with spinal cord injuries. Am J Phys Med Rehabil. 2007; 86 (2): 104-13.

[13] Kirshblum S, Waring W 3rd. Updates for the International Standards for Neurological Classification of Spinal Cord Injury. Phys Med Rehabil Clin N Am. 2014; 25: 505-517.

[14] Tzanos IA, Mavrogenis A, Gioti K, Papagelopoulos P, Panagiotopoulos E. Depressive mood in individuals with spinal cord injury (SCI) living in Greece. Spinal Cord. 2018 Sep; 56 (9): 883-889. 
[15] Kyriakides A. The development of the first Greek Spinal Cord unit. Past-Present-Future. The Rio SCI Research Project. European Journal of Physical and Rehabilitation Medicine 2016; 52 (suppl 1, pt 1): 301.

[16] Levi R, Hultling C, Seiger A. The Stockholm Spinal Cord Injury Study. 3. Health-related issues of the Swedish annual level-of-living survey in SCI subjects and controls. Paraplegia. 1995; 33 (12): 726-30.

[17] Holicky R, Charlifue S. Ageing with spinal cord injury: the impact of spousal support. Disabil Rehabil. 1999; 21 (5-6): 250-7.

[18] Putzke JD, Elliott TR, Scott Richards J. Marital Status and Adjustment 1 Year Post-Spinal-Cord-Injury. J Clin Psychol Med Sett. 2001; 8 (2): 101-7.

[19] Dijkers MP. Correlates of life satisfaction among persons with spinal cord injury. Arch Phys Med Rehabil. 1999; 80 (8): 86776.

[20] Ramakrishnan K, Mazlan M, Julia PE, Abdul Latif L. Return to work after spinal cord injury: factors related to time to first job. Spinal Cord. 2011; 49 (8): 924-7.

[21] Geyh S, Nick E, Stirnimann D, Ehrat S, Michel F, Peter C, Lude P. Self-efficacy and self-esteem as predictors of participation in spinal cord injury-an ICF-based study. Spinal Cord. 2012; 50 (9): 699-706.

[22] Celik B, Ones K, Celik EC, Bugdayci DS, Paker N, Avci C, Ince N. The effects of using the Internet on the health-related quality of life in people with spinal cord injury: a controlled study. Spinal Cord. 2014; 52 (5): 388-91.

[23] McColl MA, Arnold R, Charlifue S, Glass C, Savic G, Frankel H. Aging, spinal cord injury, and quality of life: structural relationships. Arch Phys Med Rehabil. 2003; 84 (8): 1137-44.

[24] Mathew A, Samuelkamaleshkumar S, Radhika S, Elango A. Engagement in occupational activities and pressure ulcer development in rehabilitated South Indian persons with spinal cord injury. Spinal Cord. 2013; 51 (2): 150-5.

[25] Blanes L, Carmagnani MI, Ferreira LM. Quality of life and self-esteem of Persons with paraplegia living in São Paulo, Brazil. Qual Life Res. 2009; 18 (1): 15-21.

[26] Lourenco L, Blanes L, Salomé GM, Ferreira LM. Quality of life and self-esteem in patients with paraplegia and pressure ulcers: a controlled cross-sectional study. J Wound Care. 2014; 23 (6): 331-334 336-7.

[27] Jensen MP, Hoffman AJ, Cardenas DD. Chronic pain in individuals with spinal cord injury: a survey and longitudinal study. Spinal Cord. 2005; 43: 704-12.

[28] Hartoonian N, Hoffman JM, Kalpakjian CZ. Taylor HB, Krause JK, Bombardier CH. Evaluating a spinal cord injuryspecific model of depression and quality of life. Arch Phys Med Rehabil. 2014; 95 (3): 455-65.

[29] Burke D, Lennon O, Fullen BM Quality of life after spinal cord injury: The impact of pain. Eur J Pain. 2018 Oct; 22 (9): 1662-1672. 